\title{
Modeling, Design, and Verification of a Burner for Partial Oxidation of Biomass Product Gas in an Autothermal Reformer
}

Zahra S. Musavi*, Lars J. Pettersson, Klas Engvall

Department of Chemical Engineering and Technology, KTH Royal Institute of Technology, S-100 44 Stockholm, Sweden

E-mail:*musavi@kth.se

\section{SUPPORTING INFORMATION}

1. Inputs to the Model and Description of the Settings

2. Supplementary Modeling Results with Additional Burner Geometries

3. The Gasifier Conditions in the Experiments with Real Raw Gas 


\section{Inputs to the Model and Description of the Settings}

A nonpremixed combustion kinetics system that is built into the Fluent was used; the oxidizer and the raw gas fuels were mixed in the nozzle of the burner. A couple of assumptions and settings were considered during modeling: Non-adiabatic and turbulent fluctuations, equal diffusion coefficients for all species and enthalpy (turbulent flow is assumed), chemical equilibrium, and that all thermochemical scalars were related to the average mixture. Some of these settings are grouped under the composition PDF transport model in the ANSYS programming products. The PDF transport modeling is based on finite-rate chemistry in turbulent flames. It also allows predicting and considering the intermediate species during the partial combustion.

Standard k- $\varepsilon$ was applied for viscosity and a simple case of the radiation model, 'Differential Approximation' or P1, was used for the radiation effects. All walls were considered as grey/opaque and diffusing. The $\mathrm{P} 1$ radiation model is a simple case of the more general P-N model. This radiation model is easy to use and works reasonably well for the combustion. Furthermore, it could easily be applied to complicated geometries, there may, however, be a loss of accuracy, depending on the complexity of the geometry. The Discrete Ordinates (DO) model would be a better model since it also considers the reflective index for the semi-transparent media and more radiation properties of the solid materials.

The P1 model requires entering the absorption coefficient of the flowing gas and the internal emissivity of the grey walls. The scattering coefficient of the gas was set to zero since there were no particles considered to exist in the gas. The absorption coefficient was calculated from the gas mixture as the WSGGM model (the weighted-sum-of-grey-gases model). Its value was in the range 0.64 to $2.63\left[\mathrm{~m}^{-1}\right]$ for the combustion zone. The internal emissivity of grey walls could be set to a value between 0.35 and 0.8 according to the different wall materials. For refractory materials the internal emissivity of 0.38 could be used. For Inconel sheets it is around $0.28-0.58$ and for polished Inconel sheets a lower value of $0.19-0.21$ is valid

Inputs to the model are summarized in the following Table S1.

\section{Supplementary Modeling Results with Additional Burner Geometries}

Examples of geometries with different nozzle and burner configurations, considered in the evaluation. Partial combustion was modelled for four cases, two of them are shown in this supporting information. 
Table S1. Summary of the Model Inputs (Material Properties, Velocity Constants, Setting for the Walls, Radiation Settings)

\begin{tabular}{|c|c|}
\hline material properties & method/value \\
\hline density & calculated by PDF mixture \\
\hline$C_{p}$ (Specific heat) & calculated through mixing-law \\
\hline thermal conductivity $[\mathrm{W} / \mathrm{m} \cdot \mathrm{K}]$, constant & 0.0454 \\
\hline dynamic viscosity $[\mathrm{kg} / \mathrm{m} \cdot \mathrm{s}]$, constant & $1.72 \times 10^{5}$ \\
\hline \multicolumn{2}{|l|}{ Velocity model constants } \\
\hline $\mathrm{C}_{\mu}$ & 0.09 \\
\hline $\mathrm{C}_{1} \varepsilon$ & 1.44 \\
\hline $\mathrm{C}_{2} \varepsilon$ & 1.92 \\
\hline TKE Prandtl Number ${ }^{a}$ & 1 \\
\hline TDR Prandtl Number ${ }^{\mathrm{b}}$ & 1.3 \\
\hline energy Prandtl Number & 0.85 \\
\hline wall Prandtl number & 0.85 \\
\hline PDF Schmidt number $^{\mathrm{c}}$ & 0.85 \\
\hline \multicolumn{2}{|l|}{ setting for the walls } \\
\hline wall roughness constant & 0.5 \\
\hline internal emissivity of walls, constant & 0.38 \\
\hline thermal conductivity $[\mathrm{W} / \mathrm{m} \cdot \mathrm{K}]$, constant & 202.4 \\
\hline $\mathrm{Cp}[\mathrm{J} / \mathrm{kg} \cdot \mathrm{K}]$, constant & 871 \\
\hline density $\left[\mathrm{kg} / \mathrm{m}^{3}\right]$, constant & 2719 \\
\hline \multicolumn{2}{|l|}{ model radiation settings } \\
\hline scattering coefficient $\left[\mathrm{m}^{-1}\right]$ & 0 \\
\hline absorption coefficient $\left[\mathrm{m}^{-1}\right]$ & WSGGM-domain-based \\
\hline refractive index, constant & 1 \\
\hline \multicolumn{2}{|c|}{$\begin{array}{l}\text { a The effective Prandtl number for transport of turbulence kinetic energy. This } \\
\text { defines the ratio of the momentum diffusivity to the diffusivity of turbulence kinetic } \\
\text { energy via turbulent transport. }{ }^{b} \text { The effective Prandtl number for transport of the } \\
\text { turbulent dissipation rate. This defines the ratio of the momentum diffusivity to the } \\
\text { diffusivity of turbulence dissipation via turbulent transport. c The model } \\
\text { constant } \sigma_{t} \text { for transport equations of the gas mixture in non-premixed combustion }\end{array}$} \\
\hline
\end{tabular}




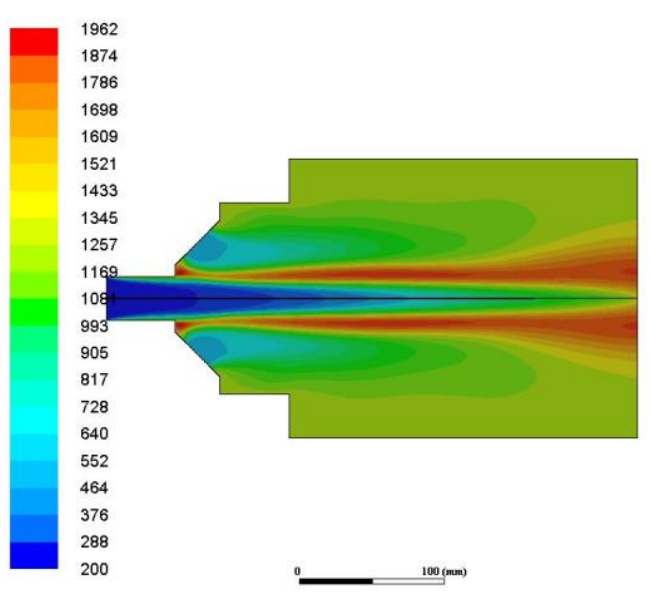

a

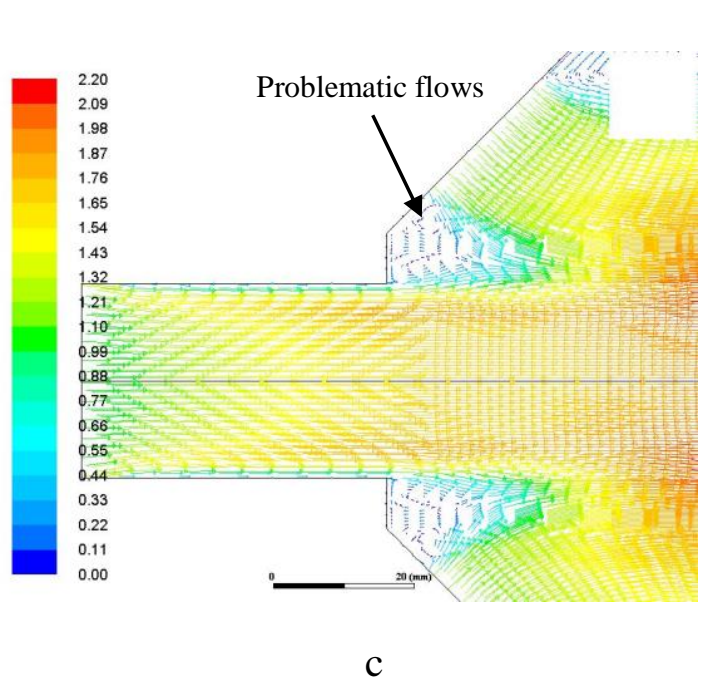

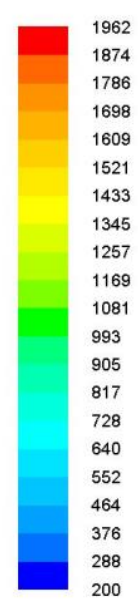

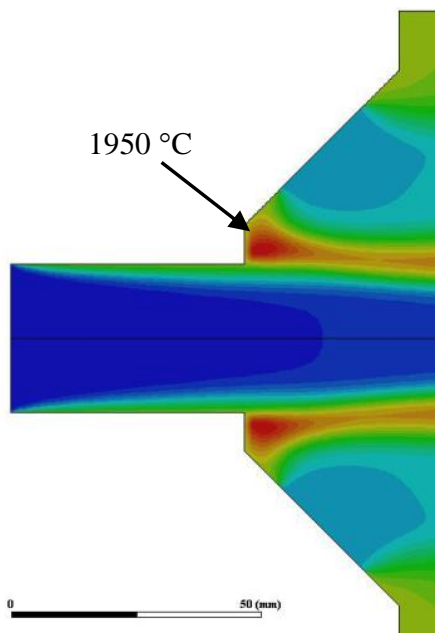

b

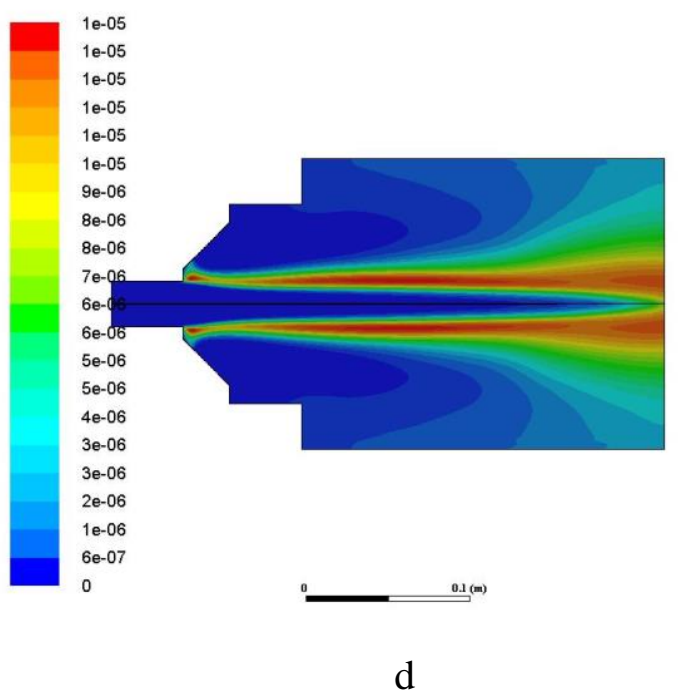

Figure S1. Modeling results with the geometry 1, (a) Contours of temperatures for the whole burner zone $\left({ }^{\circ} \mathrm{C}\right)$, (b) A zoom of contours of temperatures at the high temperature walls of a) $\left({ }^{\circ} \mathrm{C}\right)$, (c) A zoom of vectors of velocity in similar area as $(\mathrm{b})(\mathrm{m} / \mathrm{s}),(\mathrm{d})$ Contours of mole fraction of $\mathrm{HO}_{2}$ intermediate radicals. 

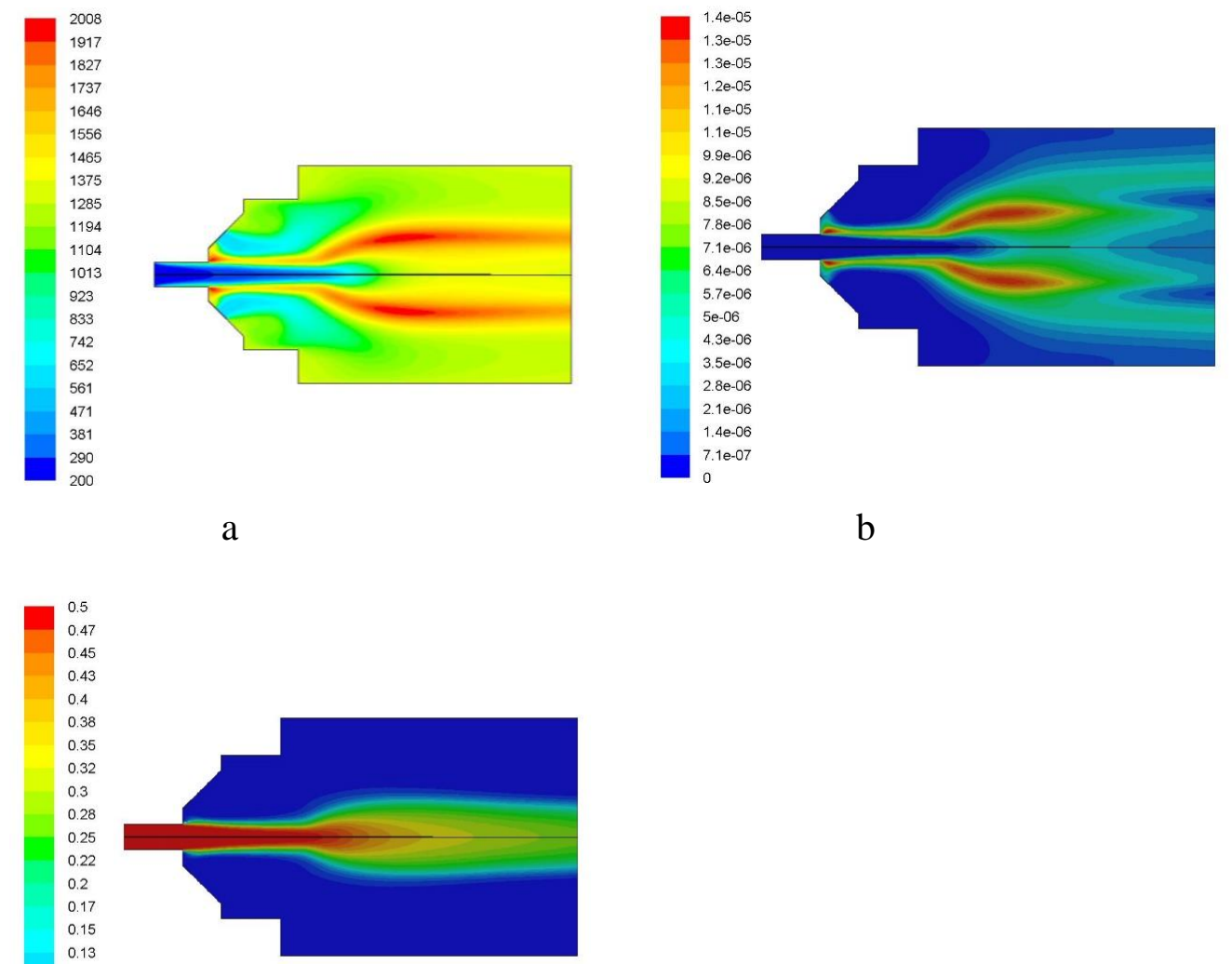

b

C

Figure S2. Modeling results with the geometry 2, (a) Contours of temperatures for the whole burner zone $\left({ }^{\circ} \mathrm{C}\right)$, (b) Contours of mole fraction of $\mathrm{HO}_{2}$ intermediate radicals, c) Contours of mole fraction of $\mathrm{O}_{2}$.

\section{The Gasifier Conditions in the Experiments with Real Raw Gas}

Table S2. Operating Data of Gasifier in the Experiments with Real Raw Gas

$\begin{array}{lc}\begin{array}{l}\text { gasifier operating data } \\ \text { uuel type }\end{array} & \text { Wood pellets } \\ \begin{array}{lc}\text { fuel moisture content } & \sim 8[\mathrm{wt} \%] \\ \text { air flow } & 43.2\left[\mathrm{Nm}^{3} / \mathrm{h}\right] \\ \text { steam flow } & 37[\mathrm{~kg} / \mathrm{h}] \\ \text { fuel feed rate } & 48-54[\mathrm{~kg} / \mathrm{h}] \\ & -3[\mathrm{hPa}] \\ \text { average gasification pressure } & 840\left[{ }^{\circ} \mathrm{C}\right]\end{array} \\ \begin{array}{ll}\text { average gasification temperature } & \text { Temperature Agent Gasification) }\end{array}\end{array}$

